\title{
BIBECHANA
}

A Multidisciplinary Journal of Science, Technology and Mathematics ISSN 2091-0762 (Print), 2382-5340 (Online)

Journal homepage: http://nepjol.info/index.php/BIBECHANA

Publisher: Research Council of Science and Technology, Biratnagar, Nepal

\section{On contact conformal curvature tensor in LP-Sasakian manifolds}

\section{Riddhi Jung Shah}

Department of Mathematics, Janata Campus, Nepal Sanskrit University, Dang, Nepal.

"Email: shahrjgeo@gmail.com

Article history: Received 01 February, 2017; Accepted 27 October, 2017

DOI: http://dx.doi.org/10.3126/bibechana.v15i0.16507

This work is licensed under the Creative Commons CC BY-NC License.

https://creativecommons.org/licenses/by-nc/4.0/

\section{(i) (9)}

Abstract

The purpose of the present paper is to study the contact conformal curvature tensor in LP-Sasakian manifolds. Some properties of contact conformally flat, $\xi$-contact conformally flat and contact conformally semi-symmetric LP-Sasakian manifolds are obtained.

Keywords: Contact conformal curvature tensor; LP-Sasakian manifold; $\eta$-Einstein manifold.

\section{Introduction}

The contact conformal curvature tensor is a curvature like tensor defined on a contact metric manifold which is constructed from the conformal curvature tensor by using the Boothby-Wang's fibration [1]. Jeong, Lee and Pak [2] defined the contact conformal curvature tensor on $(2 n+1)$-dimensional Sasakian manifolds and proved that it is invariant under D-homothetic deformation. They also proved that a Sasakian manifold $M^{2 n+1}(n>2)$ with vanishing contact conformal curvature tensor field is of constant $\varphi$ -homothetic sectional curvature $[r-n(3 n+1)] / n(n+1)$. Pak and Shin [3] gave a geometric characterization of a contact metric manifold with vanishing contact conformal curvature tensor by showing that for $n>2$, every $(2 n+1)$-dimensional contact metric manifold with vanishing contact conformal curvature tensor is a Sasakian space form. Bang and Kye [4] studied contact conformal curvature tensor on 3-dimensional Sasakian manifolds and gave a partial extension of Pak and Shin's result to 3-dimensional locally $\varphi$-symmetric contact metric manifold and also showed that the contact conformal curvature tensor on 3-dimensional Sasakian manifold always vanishes. On the other hand, Matsumoto [5] introduced the notion of Lorentzian para-Sasakian manifold. Then Mihai and Rosca [6] introduced the same notion independently and obtained many results on this manifold. Lorentzian para-Sasakian manifolds have also been studied by Matsumoto and Mihai [7], De et al. [8], Shaikh and Biswas [9] and Bagewadi et al. [10].

\section{Preliminaries}

A differentiable manifold of dimension $(2 n+1)$ is called Lorentzian para-Sasakian manifold (briefly, LPSasakian manifold) if it admits a $(1,1)$ tensor field $\varphi$, a contravariant vector field $\xi$, a 1 -form $\eta$ and a Lorentzian metric $g$ which satisfy 


$$
\begin{gathered}
\eta(\xi)=-1, \varphi^{2}(X)=X+\eta(X) \xi \\
g(\varphi X, \varphi Y)=g(X, Y)+\eta(X) \eta(Y), \\
g(X, \xi)=\eta(X), \\
\nabla_{X} \xi=\varphi X, \\
\left(\nabla_{X} \varphi\right) Y=g(X, Y) \xi+\eta(Y) X+2 \eta(X) \eta(Y) \xi
\end{gathered}
$$

where $\nabla$ denotes the covariant differentiation with respect to the Lorentzian metric $g[5,7]$.

In an LP-Sasakian manifold it can be easily seen that;

$$
\begin{gathered}
\varphi \xi=0, \eta \circ \varphi=0, \operatorname{rank} \varphi=2 n . \text { If we put } \\
\Phi(X, Y)=g(X, \varphi Y),
\end{gathered}
$$

for any vector fields $X$ and $Y$, then the tensor field $\Phi(X, Y)$ is a symmetric $(0,2)$ tensor field [5]. Also since the 1-form $\eta$ is closed in an LP-Sasakian manifold we have

$$
\left(\nabla_{X} \eta\right)(Y)=\Phi(X, Y)=g(X, \varphi Y)=g(\varphi X, Y), \Phi(X, \xi)=0
$$

for any vector fields $X$ and $Y$ [5,9]. An LP-Sasakian manifold $M$ is said to be $\eta$-Einstein if its Ricci tensor $S$ of type $(0,2)$ is of the form

$$
S(X, Y)=a g(X, Y)+b \eta(X) \eta(Y)
$$

for any vector fields $X$ and $Y$, where $a, b$ are smooth functions on the manifold. In particular, if $b=0$, then the manifold is said to be an Einstein manifold. In a $(2 n+1)$-dimensional LP-Sasakian manifold the following relations hold:

$$
\begin{gathered}
\eta(R(X, Y) Z)=g(Y, Z) \eta(X)-g(X, Z) \eta(Y), \\
R(\xi, X) Y=g(X, Y) \xi-\eta(Y) X \\
R(X, Y) \xi=\eta(Y) X-\eta(X) Y \\
R(\xi, X) \xi=X+\eta(X) \xi \\
S(X, \xi)=2 n \eta(X) \\
S(\varphi X, \varphi Y)=S(X, Y)+2 n \eta(X) \eta(Y)
\end{gathered}
$$

for any vector fields $X, Y$ and $Z$, where $R$ and $S$ are the Riemannian curvature tensor and Ricci tensor of the manifold, respectively $[8,9]$. In a $(2 n+1)$-dimensional LP-Sasakian manifold the contact conformal curvature tensor $C_{0}$ of type $(1,3)$ is defined by [2] can be written as

$$
\begin{aligned}
C_{0}(X, Y) Z= & R(X, Y) Z+\frac{1}{2 n}\{S(Y, Z) X-S(X, Z) Y+g(Y, Z) Q X \\
& -g(X, Z) Q Y+\eta(Y) S(X, Z) \xi-\eta(X) S(Y, Z) \xi+\eta(X) \eta(Z) Q Y \\
& -\eta(Y) \eta(Z) Q X+S(\varphi X, Z) \varphi Y-S(\varphi Y, Z) \varphi X+g(\varphi X, Z) Q(\varphi Y) \\
& -g(\varphi Y, Z) Q(\varphi X)+2 g(\varphi X, Y) Q(\varphi Z)+2 S(\varphi X, Y) \varphi Z\} \\
& +\frac{1}{2 n(n+1)}\left\{2 n^{2}-n-2+\frac{(n+2) r}{2 n}\right\}\{g(\varphi Y, Z) \varphi X-g(\varphi X, Z) \varphi Y \\
& -2 g(\varphi X, Y) \varphi Z\}+\frac{1}{2 n(n+1)}\left\{n+2-\frac{(3 n+2) r}{2 n}\right\}\{g(Y, Z) X \\
& -g(X, Z) Y\}-\frac{1}{2 n(n+1)}\left\{4 n^{2}+5 n+2-\frac{(3 n+2) r}{2 n}\right\}\{\eta(Y) \eta(Z) X \\
& -\eta(X) \eta(Z) Y+\eta(X) g(Y, Z) \xi-\eta(Y) g(X, Z) \xi\},
\end{aligned}
$$


where $R, S, Q$ and $r$ denote the curvature tensor, the Ricci tensor, the Ricci operator and the scalar curvature, respectively.

Definition 2.1 A $(2 n+1)$-dimensional LP-Sasakian manifold $M$ is said to be contact conformally flat if the condition

$$
C_{0}(X, Y) Z=0
$$

holds.

Definition 2.2 A $(2 n+1)$-dimensional LP-Sasakian manifold $M$ is said to be $\xi$-contact conformally flat if

$$
C_{0}(X, Y) \xi=0 .
$$

Definition 2.3 A Riemannian or pseudo-Riemannian manifold is said to be semi-symmetric if the condition

$$
R(X, Y) \cdot R=0
$$

holds, where $R(X, Y)$ denotes the derivation in the tensor algebra at each point of the manifold.

Definition 2.4 A $(2 n+1)$-dimensional LP-Sasakian manifold $M$ is said to be contact conformally semi-symmetric if

$$
R(X, Y) \cdot C_{0}=0
$$

\section{Results and Discussion}

We prove the following results which are related with above definitions

Theorem 3.1 A contact conformally flat LP-Sasakian manifold $M$ of dimension $(2 n+1)$ is an $\eta$ Einstein manifold.

Proof: Let us consider a contact conformally flat LP-Sasakian manifold $M$, then (2.17) holds and from (2.16) we have

$$
\begin{aligned}
0=R(X, Y) Z & +\frac{1}{2 n}\{S(Y, Z) X-S(X, Z) Y+g(Y, Z) Q X \\
& -g(X, Z) Q Y+\eta(Y) S(X, Z) \xi-\eta(X) S(Y, Z) \xi+\eta(X) \eta(Z) Q Y \\
& -\eta(Y) \eta(Z) Q X+S(\varphi X, Z) \varphi Y-S(\varphi Y, Z) \varphi X+g(\varphi X, Z) Q(\varphi Y) \\
& -g(\varphi Y, Z) Q(\varphi X)+2 g(\varphi X, Y) Q(\varphi Z)+2 S(\varphi X, Y) \varphi Z\} \\
& +\frac{1}{2 n(n+1)}\left\{2 n^{2}-n-2+\frac{(n+2) r}{2 n}\right\}\{g(\varphi Y, Z) \varphi X-g(\varphi X, Z) \varphi Y \\
& -2 g(\varphi X, Y) \varphi Z\}+\frac{1}{2 n(n+1)}\left\{n+2-\frac{(3 n+2) r}{2 n}\right\}\{g(Y, Z) X \\
& -g(X, Z) Y\}-\frac{1}{2 n(n+1)}\left\{4 n^{2}+5 n+2-\frac{(3 n+2) r}{2 n}\right\}\{\eta(Y) \eta(Z) X \\
- & \eta(X) \eta(Z) Y+\eta(X) g(Y, Z) \xi-\eta(Y) g(X, Z) \xi\} .
\end{aligned}
$$

Taking inner product on both sides of (3.1) by $W$, we get 
(3.2)

$$
\begin{aligned}
0= & \tilde{R}(X, Y, Z, W)+\frac{1}{2 n}\{S(Y, Z) g(X, W)-S(X, Z) g(Y, W)+g(Y, Z) S(X, W) \\
& -g(X, Z) S(Y, W)+\eta(Y) S(X, Z) \eta(W)-\eta(X) S(Y, Z) \eta(W)+\eta(X) \eta(Z) S(Y, W) \\
& -\eta(Y) \eta(Z) S(X, W)+S(\varphi X, Z) g(\varphi Y, W)-S(\varphi Y, Z) g(\varphi X, W)+g(\varphi X, Z) S(\varphi Y, W) \\
& -g(\varphi Y, Z) S(\varphi X, W)+2 g(\varphi X, Y) S(\varphi Z, W)+2 S(\varphi X, Y) g(\varphi Z, W)\} \\
& +\frac{1}{2 n(n+1)}\left\{2 n^{2}-n-2+\frac{(n+2) r}{2 n}\right\}\{g(\varphi Y, Z) g(\varphi X, W)-g(\varphi X, Z) g(\varphi Y, W) \\
- & 2 g(\varphi X, Y) g(\varphi Z, W)\}+\frac{1}{2 n(n+1)}\left\{n+2-\frac{(3 n+2) r}{2 n}\right\}\{g(Y, Z) g(X, W) \\
- & g(X, Z) g(Y, W)\}-\frac{1}{2 n(n+1)}\left\{4 n^{2}+5 n+2-\frac{(3 n+2) r}{2 n}\right\}\{\eta(Y) \eta(Z) g(X, W) \\
- & \eta(X) \eta(Z) g(Y, W)+\eta(X) g(Y, Z) \eta(W)-\eta(Y) g(X, Z) \eta(W)\},
\end{aligned}
$$

where $\tilde{R}(X, Y, Z, W)=g(R(X, Y) Z, W)$. Setting $W=\xi$ in (3.2) and using (2.1), (2.3), (2.6), (2.10), (2.14) and then further simplifying yields

$$
\begin{aligned}
0= & \left\{\frac{8 n^{3}+10 n^{2}+4 n-(3 n+2 r)}{2 n(2 n+1)}\right\}\{g(Y, Z) \eta(X)-g(X, Z) \eta(Y)\} \\
& +S(Y, Z) \eta(X)-S(X, Z) \eta(Y) .
\end{aligned}
$$

In (3.3) replacing $X$ by $\xi$ and using (2.1), (2.3) and (2.14), we get

$$
\begin{aligned}
S(Y, Z)= & \left\{-\frac{2 n\left(4 n^{2}+5 n+2\right)-(3 n+2) r}{2 n(n+1)}\right\} g(Y, Z) \\
& +\left\{-\frac{2 n\left(6 n^{2}+7 n+2\right)-(3 n+2) r}{2 n(n+1)}\right\} \eta(Y) \eta(Z) .
\end{aligned}
$$

Equation (3.4) implies that

$$
S(Y, Z)=\alpha g(Y, Z)+\beta \eta(Y) \eta(Z)
$$

where $\alpha=-\frac{2 n\left(4 n^{2}+5 n+2\right)-(3 n+2) r}{2 n(n+1)}$ and $\beta=-\frac{2 n\left(6 n^{2}+7 n+2\right)-(3 n+2) r}{2 n(n+1)}$. The relation (3.5) implies that the manifold is an $\eta$-Einstein manifold. This completes the proof of the theorem.

Theorem 3.2 Let $M$ be a $(2 n+1)$-dimensional LP-Sasakian manifold. If the condition $C_{0}(X, Y) \xi=0$ holds in $M$, then the manifold is an $\eta$-Einstein manifold.

Proof: Let us consider a $(2 n+1)$-dimensional LP-Sasakian manifold $M$ which is $\xi$-contact conformally flat, then we have $C_{0}(X, Y) \xi=0$. Now, replacing $Z$ by $\xi$ in (2.16) and using (2.1), (2.3), (2.6), (2.12), (2.14) and (2.18), we get

$$
\begin{aligned}
0= & \left\{\frac{2 n\left(4 n^{2}+5 n+2\right)-(3 n+2) r}{2 n(n+1)}\right\}\{\eta(Y) X-\eta(X) Y\} \\
& +\eta(Y) Q X-\eta(X) Q Y .
\end{aligned}
$$

Taking inner product on both sides of (3.6) by $W$, we obtain 


$$
\begin{aligned}
S(Y, W) \eta(X)= & \left\{\frac{2 n\left(4 n^{2}+5 n+2\right)-(3 n+2) r}{2 n(n+1)}\right\}\{g(X, W) \eta(Y)-g(Y, W) \eta(X)\} \\
& +S(X, W) \eta(Y) .
\end{aligned}
$$

Putting $X=\xi$ in (3.7) and using (2.1), (2.3) and (2.14), we get

$$
\begin{aligned}
S(Y, W)= & \left\{-\frac{2 n\left(4 n^{2}+5 n+2\right)-(3 n+2) r}{2 n(n+1)}\right\} g(Y, W) \\
& +\left\{-\frac{2 n\left(6 n^{2}+7 n+2\right)-(3 n+2) r}{2 n(n+1)}\right\} \eta(Y) \eta(W) .
\end{aligned}
$$

From (3.8), we have

$$
S(Y, W)=A g(Y, W)+B \eta(Y) \eta(W),
$$

where $A=\left\{-\frac{2 n\left(4 n^{2}+5 n+2\right)-(3 n+2) r}{2 n(n+1)}\right\}$ and $B=\left\{-\frac{2 n\left(6 n^{2}+7 n+2\right)-(3 n+2) r}{2 n(n+1)}\right\}$. Hence the manifold is an $\eta$-Einstein manifold. This completes the proof of the theorem.

Theorem 3.3 A contact conformally semi-symmetric LP-Sasakian manifold $\left(M^{2 n+1}, g\right)$ is an Einstein manifold and a manifold of constant curvature $r=2 n(2 n+1)$.

Proof: Let us consider an LP-Sasakian manifold $\left(M^{2 n+1}, g\right)$ satisfying the condition $R(X, Y) \cdot C_{0}=0$. Now, we have

$$
\begin{aligned}
\left(R(X, Y) \cdot C_{0}\right)(U, V) Z= & R(X, Y) C_{0}(U, V) Z-C_{0}(R(X, Y) U, V) Z \\
& -C_{0}(U, R(X, Y) V) Z-C_{0}(U, V) R(X, Y) Z .
\end{aligned}
$$

In view of (2.20) and (3.10), we get

$$
\begin{aligned}
0= & R(X, Y) C_{0}(U, V) Z-C_{0}(R(X, Y) U, V) Z \\
& -C_{0}(U, R(X, Y) V) Z-C_{0}(U, V) R(X, Y) Z .
\end{aligned}
$$

Taking $X=\xi$ in (3.11) and using (2.11), we obtain

$$
\begin{aligned}
0= & g\left(C_{0}(U, V) Z, Y\right) \xi-\eta\left(C_{0}(U, V) Z\right) Y-g(Y, U) C_{0}(\xi, V) Z \\
& +\eta(U) C_{0}(Y, V) Z-g(Y, V) C_{0}(U, \xi) Z+\eta(V) C_{0}(U, Y) Z \\
& -g(Y, Z) C_{0}(U, V) \xi+\eta(Z) C_{0}(U, V) Y .
\end{aligned}
$$

Taking inner product on both sides of (3.12) by $\xi$, we get

$$
\begin{aligned}
0= & -g\left(C_{0}(U, V) Z, Y\right)-\eta\left(C_{0}(U, V) Z\right) \eta(Y)-g(Y, U) \eta\left(C_{0}(\xi, V) Z\right) \\
& +\eta(U) \eta\left(C_{0}(Y, V) Z\right)-g(Y, V) \eta\left(C_{0}(U, \xi) Z\right)+\eta(V) \eta\left(C_{0}(U, Y) Z\right) \\
& -g(Y, Z) \eta\left(C_{0}(U, V) \xi\right)+\eta(Z) \eta\left(C_{0}(U, V) Y\right) .
\end{aligned}
$$

Putting $Y=U$ in (3.13) we obtain

$$
\begin{aligned}
& 0=g\left(C_{0}(U, V) Z, U\right)+g(U, U) \eta\left(C_{0}(\xi, V) Z\right)+g(U, V) \eta\left(C_{0}(U, \xi) Z\right) \\
& -\eta(V) \eta\left(C_{0}(U, U) Z\right)+g(U, Z) \eta\left(C_{0}(U, V) \xi\right)-\eta(Z) \eta\left(C_{0}(U, V) U\right) .
\end{aligned}
$$

Now, from (2.16) we have

$$
\eta\left(C_{0}(U, V) \xi\right)=0
$$




$$
\begin{aligned}
\eta\left(C_{0}(\xi, V) Z\right)= & -\frac{1}{n} S(V, Z)-\frac{2 n\left(4 n^{2}+5 n+2\right)-(3 n+2) r}{2 n^{2}(n+1)} g(V, Z) \\
& -\frac{2 n\left(6 n^{2}+7 n+2\right)-(3 n+2) r}{2 n^{2}(n+1)} \eta(V) \eta(Z)
\end{aligned}
$$

and

$$
\eta\left(C_{0}(U, U) Z\right)=0
$$

By virtue of (3.15) and (3.17), (3.14) reduces to

$$
\begin{aligned}
0= & g\left(C_{0}(U, V) Z, U\right)+g(U, U) \eta\left(C_{0}(\xi, V) Z\right) \\
& +g(U, V) \eta\left(C_{0}(U, \xi) Z\right)-\eta(Z) \eta\left(C_{0}(U, V) U\right) .
\end{aligned}
$$

Let $\left\{e_{i}: i=1,2, . ., 2 n+1\right\}$ be an orthonormal basis of the tangent space at any point of the manifold. Putting $U=e_{i}$ in (3.18) and taking summation over $i, 1 \leq i \leq 2 n+1$, we get

$$
\sum_{i=1}^{2 n+1} g\left(C_{0}\left(e_{i}, V\right) Z, e_{i}\right)+2 n \eta\left(C_{0}(\xi, V) Z\right)-\sum_{i=1}^{2 n+1} \eta(Z) \eta\left(C_{0}\left(e_{i}, V\right) e_{i}\right)=0
$$

since $\sum_{i=1}^{2 n+1} g\left(e_{i}, V\right) \eta\left(C_{0}\left(e_{i}, \xi\right) Z\right)=-\eta\left(C_{0}(\xi, V) Z\right)$.

Again, from (2.16) it follows

$$
\begin{aligned}
& \sum_{i=1}^{2 n+1} g\left(C_{0}\left(e_{i}, V\right) Z, e_{i}\right) \\
& =\frac{2(n+1)}{n} S(V, Z)+\left[-\frac{2 n\left(4 n^{2}-n-2\right)+\left(2 n^{2}+n+2\right) r}{2 n^{2}(n+1)}\right] g(V, Z) \\
& +\left[-\frac{4 n\left(2 n^{3}-n^{2}-5 n-2\right)+\left(-2 n^{2}+2 n+4\right) r}{2 n^{2}(n+1)}\right] \eta(V) \eta(Z)
\end{aligned}
$$

under the condition $\operatorname{tr} . \varphi=\operatorname{tr} .(\varphi Q)=0$ and by the use of (2.2), (2.8) and (2.15). From the definition of contact conformal curvature tensor, we also have

$$
\sum_{i=1}^{2 n+1} \eta(Z) \eta\left(C_{0}\left(e_{i}, V\right) e_{i}\right)=\frac{(2 n+1)\{r-2 n(2 n+1)\}}{n(n+1)} \eta(V) \eta(Z) .
$$

In view of (3.16), (3.20) and (3.21), (3.21) takes the form

(3.22) $\quad S(V, Z)=\lambda_{1} g(V, Z)+\lambda_{2} \eta(V) \eta(Z)$,

where $\lambda_{1}=\frac{2 n\left(8 n^{3}+14 n^{2}+3 n-2\right)-\left(4 n^{2}+3 n-2\right) r}{4 n(n+1)}$ and $\lambda_{2}=\frac{(n-1)\{2 n(2 n+1)-r\}}{n}$.

Taking an orthonormal frame field at any point of the manifold and contracting over $V$ and $Z$ in (3.22) we get

Using (3.23) in (3.22) we obtain

$$
r=2 n(2 n+1)
$$

$$
S(V, Z)=2 n g(V, Z)
$$

In view of (3.23) and (3.24), the theorem is proved.

\section{Conclusions}

In this paper, we have studied on contact conformal curvature tensor in a $(2 n+1)$-dimensional Lorentzian para-Sasakian manifold (briefly, LP-Sasakian manifold). We have investigated that 
contact conformally flat and $\xi$-contact conformally flat LP-Sasakian manifold is an $\eta$-Einstein manifold. It is also proved that a contact conformally semi-symmetric LP-Sasakian manifold is an Einstein manifold.

\section{Acknowledgement}

The author is grateful to worthy referee for his/her valuable suggestions and comments for the improvement of this paper.

\section{References}

[1] W. M. Boothby, H. C. Wang, On contact manifolds, Ann. of Math. 68 (1958) 721-734. doi.org/10.2307/1970165.

[2] J .C. Jeong, J.D. Lee, G.H. Oh, J.S. Pak, On the contact conformal curvature tensor, Bull. Korean Math. Soc. 27 (1990) 133-142.

[3] J.S. Pak, Y.J. Shin, A note on contact conformal curvature tensor, Comm. Korean Math. Soc. 13(2) (1998) 337-343.

[4] K. Bang, J.Y. Kye, Vanishing of contact conformal curvature tensor on 3-dimensional Sasakian manifolds, Kangweon-kyngki Math. J. 10 (2) (2002) 157-166.

[5] K. Matsumoto, On Lorentzian paracontact manifolds, Bull. of Yamagata Univ. Nat. Sci. 12 (1989) 151156.

[6] I. Mihai, R. Rosca, On Lorentzian P-Sasakian manifolds, Classical Analysis, World. Scientific Publi. (1992) 155-169.

[7] K. Matsumoto, I. Mihai, On a certain transformation in a Lorentzian para-Sasakian manifold, Tensor N. S. 47 (1988), 189-197.

[8] U. C. De, K. Matsumoto, A. A. Shaikh, On Lorentzian para-Sasakian manifolds, Rendiconti del Seminario Matematico di Mesina, Serrie II, Supplemento al n. 3 (1999) 149-158.

[9] A. A. Shaikh, S. Biswas, On LP-Sasakian manifolds, Bull. Malaysian Sci. Soc. 27 (2007) 17-26.

[10] C. S. Bagewadi, Venkatesha, N.S. Basavarajappa, On LP-Sasakian manifolds, Scientia Series A, Mathematical Sciences 16 (2008) 1-8.

[11] Z. I. Szabo, Structures theorems on Riemannian spaces satisfying, I, J. Diiff. Geom. 17 (1982) 531-582. 